Gynäkologische Endokrinologie 2015 · 13:5-6 DOI 10.1007/s10304-015-0696-9

Online publiziert: 4. Februar 2015

c) Springer-Verlag Berlin Heidelberg 2015

\author{
M. Birkhäuser ${ }^{1} \cdot$ T. Strowitzki ${ }^{2}$ \\ ${ }^{1}$ Basel \\ ${ }^{2}$ Gynäkologische Endokrinologie und Fertilitätsstörungen, Universitäts-Frauenklinik, Heidelberg
}

\title{
Knochen und Gelenke
}

Die Osteoporose ist eine Volkskrankheit. Sie ist durch eine verminderte Knochenmasse und gestörte Knochenarchitektur charakterisiert. Die hauptsächliche klinische Konsequenz der Erkrankung ist eine Erhöhung des Risikos für Fragilitätsfrakturen. Fragilitätsfrakturen sind mit starken Schmerzen, Leiden, Behinderungen und $\mathrm{u}$. U. mit bleibender Invalidität oder gar dem Tod verbunden. Zudem entstehen der Gesellschaft durch osteoporotische Frakturen erhebliche Kosten. Allein für die Schweiz betragen diese 2,050 Mrd. Franken pro Jahr. Laut Schätzungen werden sie bis zum Jahr 2025 noch um 29\% ansteigen. Die Osteoporose gehört somit zu den größten Kostenfaktoren im Gesundheitswesen überhaupt. Bei der Frau sind die Kosten einer Akuthospitalisation wegen osteoporotischer Frakturen ungefähr gleich hoch wie die Kosten für kardiovaskuläre Erkrankungen und Brustkrebs zusammengenommen. Es ist daher entscheidend, das Frakturrisiko zu senken, wobei einer gezielten und wirksamen Prävention von Osteoporose und Fragilitätsfrakturen die größte Bedeutung zukommt.

Die vorliegende Ausgabe von Gynäkologische Endokrinologie geht auf einige oft verkannte Aspekte der Osteoporose und Frakturprävention bei Frauen ein.

Schwangerschaft und Stillzeit stellen eine Herausforderung für den mütterlichen Kalzium- und Knochenstoffwechsel dar, die weit unterschätzt wird. C. Kasperk fasst in seinem Beitrag zusammen, was zu dieser Thematik bekannt ist und wie negative Folgen auf den Knochenstoffwechsel der Mutter verhindert werden können.

J.D. Ringe u. E. Windler analysieren Nutzen und Risiken einer Kalzium- und Vitamin-D-Substitution beim Erwachsenen. Dabei gehen sie speziell auf den älteren Menschen ein. Sie berücksichtigen in ihrer Übersicht auch die nichtskeletalen Vorteile und Risiken einer Kalzium- und Vitamin-D-Gabe. Da zu diesem Punkt widersprüchliche Studienergebnisse vorliegen, wird eine regelmäßige moderate Substitution beider Substanzen empfohlen. Nach Angaben der Autoren bedeutet dies eine Kalziumzufuhr von 1000$1500 \mathrm{mg}$ sowie eine Vitamin-D-Einnahme von 800-2000 IE pro Tag. Bei der Vitamin-D-Substitution sind für 25-Hydroxy-Vitamin D als Verlaufsparameter Serumwerte von 30-50 $\mathrm{ng} / \mathrm{ml}$ anzustreben.

\) Das Frakturrisiko hängt nicht allein von der Knochenmasse ab

R. Theiler u. M. Kränzlin betonen in ihrem Beitrag, dass das Frakturrisiko nicht allein von der Knochenmasse abhängig ist, sondern auch von zahlreichen anderen Risikofaktoren, die durch die Knochendichtemessung nicht erfasst werden können. Deshalb ist die Evaluation des individuellen Frakturrisikos mit dem Fracture Risk Assessment Tool (FRAX ${ }^{\circledR}$ ) besser geeignet als die Knochendichtemessung allein. FRAX $^{\circledast}$ erlaubt eine Voraussage des absoluten individuellen 10-Jahres-Risikos von Frakturen des proximalen Femurs und allen weiteren wichtigen osteoporotischen Frakturen. Die Autoren weisen aber auch auf einige Probleme von FRAX ${ }^{\odot}$ hin, an denen noch gearbeitet werden muss.

M. Gambacciani belegt die Bedeutung der Menopause für Knorpel und Chondrozyten. Ein Östrogenmangel beeinflusst beides negativ. Eine Behandlung mit Östrogenen oder mit selektiven Östrogenrezeptormodulatoren (SERM) kann daher eine günstige Wirkung auf das Bindegewebe und damit auch auf die Zwischenwirbelscheiben haben, was wiederum das Frakturrisiko vermindert. 
D. Frey fasst zusammen, wie inflammatorische Erkrankungen aus dem rheumatischen Formenkreis oft mit einer Veränderung des Knochenstoffwechsels einhergehen. Sie zeigt auf, welchen Einfluss wichtige entzündlich-rheumatische Erkrankungen auf die Osteoporose und osteoporotische Frakturen ausüben. Am besten belegt ist die erhöhte Prävalenz der Osteoporose und der Fragilitätsfrakturen für die rheumatoide Arthritis und die ankylosierende Spondylitis. Bei jeder entzündlich-rheumatischen Erkrankung sollte daher an das Vorliegen einer Osteoporose gedacht werden. Gegebenenfalls muss eine entsprechende Abklärung erfolgen.

Bis heute ist oft nicht klar, wie lange eine nichthormonale Frakturprävention durchgeführt werden muss, um eine Fraktursenkung zu erreichen. K. Lippuner fasst die aktuelle Datenlage zu einem etwaigen therapeutischen Fenster bei einer Langzeittherapie zusammen. Die Notwendigkeit eines therapeutischen Fensters hängt einerseits vom zu erwartenden Nutzen und andererseits vom Auftreten möglicher seltener Langzeitunverträglichkeiten ab, sodass die Dauer der Therapie mit einem bestimmten Präparat individuell festgelegt und mit der Patientin abgesprochen werden sollte.

Unter der Rubrik „Arzneimitteltherapie" diskutiert A.O. Mueck die komplexe Pharmakologie von Progesteron, die stark von der Applikationsform abhängt und individuell variieren kann. Diese Kenntnisse sind zu Beginn einer korrekten Hormontherapie essenziell.

Die in dieser Ausgabe vereinten Beiträge werden jedem, der an gynäkologischer Endokrinologie und postmenopausaler Osteoporose interessiert ist, wertvolle Impulse für die tägliche Praxis geben. Für die Praxis relevant ist auch die alte und heute wieder bestätigte Erkenntnis, dass bei postmenopausalen Frauen mit erhöhtem Frakturrisiko unter einem Alter von 60 Jahren oder innerhalb von 10 Jahren nach der Menopause die HT eine Therapie der ersten Wahl zur Prävention und Behandlung der postmenopausalen Osteoporose und von osteoporotischen Frakturen ist [1]. Nach neueren Daten und der sorgfältigen Reanalyse älterer Arbeiten überwiegt bei der Ga- be einer HT zur Frakturprävention innerhalb dieses günstigen Zeitfensters der Nutzen klar die Risiken. Diese Meinung vertritt auch die International Menopause Society [2].

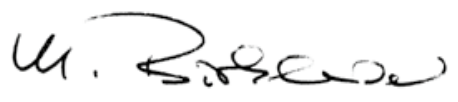

M. Birkhäuser

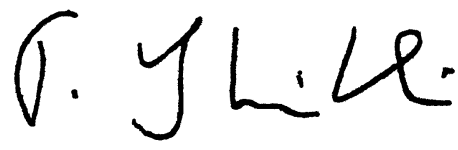

T. Strowitzki

\section{Korrespondenzadressen}

Prof. Dr. M. Birkhäuser

Gartenstr. 67, 4052 Basel

Schweiz

martin.birkhaeuser@balcab.ch

\section{Prof. Dr. T. Strowitzki}

Gynäkologische Endokrinologie und Fertilitätsstörungen, Universitäts-Frauenklinik Im Neuenheimer Feld 440, 69120 Heidelberg thomas.strowitzki@med.uni-heidelberg.de

Interessenkonflikt. M. Birkhäuser und T. Strowitzki geben an, dass kein Interessenkonflikt besteht.

\section{Literatur}

1. Birkhaeuser $M$ (2014) Primary prevention of fragility fractures in postmenopausal women (part 1): General prevention and primary prevention by MHT Ref. Gynecol Obstet 16:79-110

2. de Villiers TJ, Pines A, Panay N et al (2013) Updated 2013 International Menopause Society recommendations on menopausal hormone therapy and preventive strategies for midlife health. Climacteric 16:316-337

\section{Entwicklung von Kindern und Jugendlichen}

KiGGS: Welle 1

Die Entwicklung von Kindern und Jugendlichen sowie ihre Chancen auf ein Leben

in guter Gesundheit hängen nicht nur von körperlichen und psychischen Faktoren ab, sondern werden auch durch das familiäre und soziale Umfeld geprägt. Diese Zusammenhänge untersucht die erste Folgeerhebung (KiGGS Welle 1) der "Studie zur Gesundheit von Kindern und Jugendlichen in Deutschland" (KiGGS).

Im Schwerpunktheft „Studie zur Gesundheit von Kindern und Jugendlichen in Deutschland: Ergebnisse der ersten Folgebefragung (KiGGS:

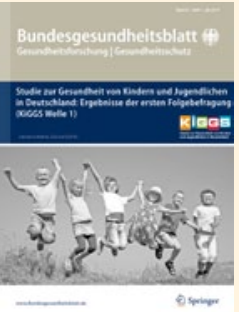
Welle 1)" (Ausgabe 7/2014) der Zeitschrift Bundesgesundheitsblatt werden u.a. folgende wichtige Daten, Analysen und Trendentwicklun-

gen aus der neuen KiGGS-Befragungswelle vorgestellt:

- Chronische Erkrankungen und impfpräventable Infektionserkrankungen

- Tabak- und Alkoholkonsum bei 11- bis 17-jährigen Jugendlichen

- Einflussfaktoren auf Verbreitung und Dauer des Stillens in Deutschland

- Körperlich-sportliche Aktivität und Nutzung elektronischer Medien im Kindesund Jugendalter

Bestellen Sie diese Ausgabe zum Preis von 16,- EUR zzgl. Versandkosten bei Springer Customer Service Center Kundenservice Zeitschriften

Haberstr. 7

69126 Heidelberg

Tel.: +49 6221-345-4303

Fax: +49 6221-345-4229

E-Mail: leserservice@springer.com

Suchen Sie noch mehr zum Thema? Mit e.Med, dem Online-Paket von Springer Medizin, können Sie schnell und komfortabel in über 600 medizinischen Fachzeitschriften recherchieren. Weitere Infos unter springermedizin.de/eMed. 